\title{
openheart Propensity score-matched analysis of patients with severe aortic stenosis undergoing surgical aortic valve replacement
}

\author{
Shiro Miura, ${ }^{\circ}$ Takehiro Yamashita, ${ }^{1}$ Michiya Hanyu, ${ }^{2}$ Hiraku Kumamaru, ${ }^{3}$ \\ Shinichi Shirai, ${ }^{4}$ Kenji Ando ${ }^{4}$
}

To cite: Miura S, Yamashita T, Hanyu M, et al. Propensity score-matched analysis of patients with severe aortic stenosis undergoing surgical aortic valve replacement. Open Heart 2019;6:e000992.

doi:10.1136/

openhrt-2018-000992

Received 12 December 2018 Revised 22 March 2019 Accepted 26 April 2019
(A) Check for updates

(c) Author(s) (or their employer(s)) 2019. Re-use permitted under CC BY-NC. No commercial re-use. See rights and permissions. Published by BMJ.

${ }^{1}$ Department of Cardiology, Hokkaido Ono Memorial Hospital, Sapporo, Japan ${ }^{2}$ Cardiovascular Centre, Tazuke Kofukai Foundation Medical Research Institute, Kitano Hospital, Osaka, Japan ${ }^{3}$ Graduate School of Medicine, The University of Tokyo, Tokyo, Japan

${ }^{4}$ Department of Cardiology, Kokura Memorial Hospital, Kitakyushu, Japan

Correspondence to Dr Shiro Miura; shirotan1027m@yahoo.co.jp

\section{ABSTRACT}

Objective Severe aortic stenosis (AS) is one of the most serious valve conditions. Patient demography and the aetiology of AS have substantially changed in the past several decades along with a drastic improvement of surgical aortic valve replacement (SAVR) and its associated procedures. Contemporary patients with severe AS have multiple comorbidities and live much longer. We aimed to elucidate the treatment effects of SAVR on long-term outcome in propensity score (PS)-matched and the entire patient populations.

Methods We retrospectively reviewed 570 patients with severe AS defined as an aortic valve area of $1.0 \mathrm{~cm}^{2}$ or less. Systemic differences in 39 baseline characteristics between non-SAVR and SAVR groups were adjusted using PS matching method. The endpoints included all-cause mortality and cardiovascular events that included heart failure, non-fatal stroke, syncope and acute coronary syndrome.

Results Overall, $55 \%$ of the entire population (mean age 78 years; males $41 \%$ ) were symptomatic. During 3.9 years of the median follow-up, $210(36 \%)$ patients underwent SAVR and 231 (41\%) died. Cumulative incidences of mortality and both mortality and cardiovascular events were significantly higher in the non-SAVR group than in the other group $(p<0.001$, each). Among 101 PS-matched pairs, SAVR correlated with a lower mortality risk (HR $0.35 ; 95 \% \mathrm{Cl} 0.21$ to $0.59 ; \mathrm{p}<0.001)$ ) and mortality and cardiovascular events combined (HR 0.62; $95 \% \mathrm{Cl} 0.42$ to $0.92 ; p=0.02)$. However, survival difference between both groups was markedly smaller among asymptomatic patients in the subgroup of matched patients.

Conclusion Patients with AS undergoing SAVR exhibit a lower incidence of all-cause mortality and major cardiovascular events than those not undergoing surgical interventions, even after the baseline characteristics are balanced by the PS matching. The correlation between SAVR and survival from cardiovascular events is less evident among asymptomatic patients.

\section{INTRODUCTION}

The prevalence of aortic stenosis (AS) increases with ageing, and $\mathrm{AS}$ is one of the leading valve abnormalities. Recently,

\section{Key questions}

What is already known about this subject?

- Among patients with severe symptomatic aortic stenosis (AS), surgical aortic valve replacement (SAVR) has been demonstrated to improve survival compared with that in unoperated patients. However, a substantial imbalance in terms of baseline characteristics, for example, comorbidities, cardiovascular risks, valve severity, performance status (frailty) and socioeconomic background, can exist between operated and unoperated patients.

What does this study add?

- In this contemporary study, patients undergoing SAVR exhibited a lower incidence of death and major cardiovascular events than medically treated patients, even after baseline characteristics were balanced via propensity score matching. However, further studies are warranted to appropriately debate the appropriate indications, methods and timing of surgical intervention for asymptomatic patients or for those with renal dysfunction.

How might this impact on clinical practice?

- These findings can have substantial implications for informed decision-making during the management of patients with severe AS among heat team members and those seeking advice on prognosis of the critical disease. Our data are also more valuable in terms of the efficacy of surgical interventions on long-term clinical outcomes over medical management in the new era of transcatheter aortic valve replacement.

degenerative AS, the most prevalent aetiology, has rapidly increased, with a prevalence of $2 \%-7 \%$ in the population aged $\geq 65$ years. ${ }^{12}$ In the past several decades, patients' profiles have shifted remarkably, with the aetiology now dominated by senile calcific AS. ${ }^{3}$ Moreover, patients are more likely to comprise marked comorbidities, such as multiple organ dysfunctions and concomitant cardiovascular diseases, all independently affecting 
mortality, as we investigated previously. ${ }^{4}$ Surgical aortic valve replacement (SAVR) is the most well-established therapy for severe AS. Besides demographic changes in patients with severe AS, remarkable advances in surgical techniques and innovative equipment have facilitated patients with operative risks to undergo surgical procedures safely.

The last two decades have witnessed the launching of minimally invasive transcatheter aortic valve replacement (TAVR) which was similar to SAVR with respect to mortality in patients with severe AS at intermediate operative risks. ${ }^{5}$ This less invasive therapy might not be a complete alternative to SAVR, as simultaneous interventions on the mitral valve, coronary artery bypass grafting (CABG), are challenging in conjunction with TAVR, and data on long-term device durability remain scarce. In the modern era when aggressive interventions are recommended to patients with severe AS, ascertaining the efficacy and safety of surgical interventions for the contemporary population of patients with severe AS remains critical, as operative risks due to age and multiple comorbidities and long-term complications, such as structural valve deterioration, prosthetic valve endocarditis and anticoagulant-induced bleeding events, in surgically treated patients remain significant. Yet there exists, for ethical reasons, a limited number of randomised controlled trials. Thus, a well-organised, observational cohort study comparing SAVR and medical management should be designed with marked differences between surgically treated and medically treated groups being appropriately adjusted for their baseline risks.

Hence, this study aims to investigate the clinical impact of SAVR compared with nonsurgical treatment on mortality and incidences of adverse cardiovascular events in patients with severe AS with adjustments by propensity score (PS) matching. Furthermore, the study assesses the benefits of SAVR in various subgroups of our interest within the matched population.

\section{METHODS}

\section{Study design and patient enrolment}

This retrospective, observational cohort study was conducted at Kokura Memorial Hospital (Kitakyushu, Japan). We reviewed the echocardiographic database for patients with severe AS who visited the hospital from August 2009 to February 2012. We defined severe AS, based on international guidelines, as an aortic valve area (AVA) of $\leq 1.0 \mathrm{~cm}^{2}$ by transthoracic echocardiography. ${ }^{6}$ Initially, we identified 595 patients from the database. Of these, 18 patients who underwent TAVR during the follow-up period and 7 patients with lost follow-up data were excluded; the remaining 570 subjects were included in the final analysis (figure 1). The study was reviewed and approved by the Institutional Review Board of Kokura Memorial Hospital, Japan (Approval number: 201402210108).

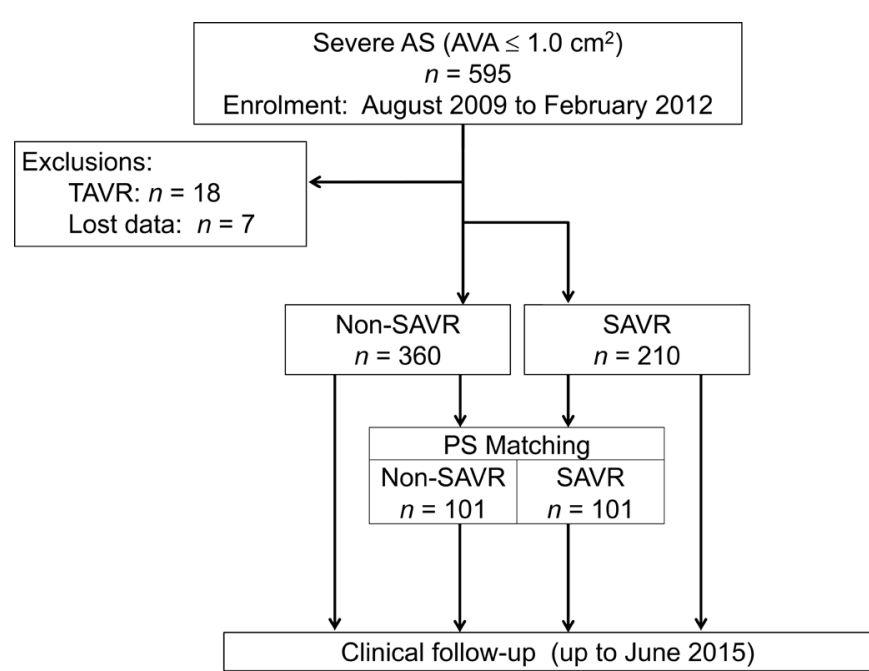

Figure 1 A flowchart of primary findings in the study population as severe aortic stenosis. AVA, aortic valve area; PS, propensity score; SAVR, surgical aortic valve replacement; TAVR, transcatheter aortic valve replacement.

\section{Baseline characteristics and echocardiographic assessment}

We collected comprehensive data, including patients' characteristics, medications and operational information from chart reviews based on the definitions provided. Activity level was defined as active if a patient lived an independent life and as limited if a patient required assisted care. Asymptomatic status was defined as no limitations of ordinary physical activity based on the New York Heart Association functional classification (NYHA class).

All patients underwent two-dimensional echocardiographic examinations, at least, once, during the study period, by professional sonographers using commercially available cardiac ultrasound machines (Vivid 7 Dimension and Vivid E9, GE Healthcare, Horten, Norway; iE33, Philips Medical Systems, Best, The Netherlands). Moreover, all patients who underwent SAVR received a preoperative echocardiographic examination within 1 month of their respective operation date. We considered these records as the baseline characteristics of those undergoing SAVR. Doppler echocardiographic measurements comprised the aortic mean pressure gradient using the simplified Bernoulli equation and an AVA using a standard continuity equation; ${ }^{7}$ the severity of aortic regurgitation, mitral regurgitation and tricuspid regurgitation was assessed per international guidelines. ${ }^{8}$

\section{Clinical follow-up and endpoints}

The primary endpoint was all-cause mortality. Cardiovascular events, including hospital admission from worsened heart failure (HF), acute coronary syndrome, syncope and non-fatal stroke, were defined as other end points. We gathered information on clinical outcome from patients, families or physicians, either in person or by telephonic interviews. We followed all patients retrospectively until June 2015 to assess their survival status. Perhaps, a time delay before a patient receives surgical treatment can account for marked survival advantages in 
the SAVR group. Hence, we defined the operation date as day 1 for the follow-up of the SAVR group, whereas the follow-up started on the day of index echocardiography (enrolment) for the non-SAVR group.

\section{Statistical analysis}

Although we compared the distributions of continuous variables using Student's $t$-test or Wilcoxon rank-sum test, $\chi^{2}$ test was used for categorical variables. Continuous data are presented as mean \pm SD for normally distributed variables or median values with 25th and 75th percentiles for non-normally distributed variables, and categorical data are presented as absolute numbers and percentages. For survival analyses in both the entire population and the matched group, the Kaplan-Meier method was used to estimate the cumulative incidence of all-cause mortality and cardiovascular events in three ways: mortality, mortality and HF requiring admission and mortality and cardiovascular events. Using the log-rank test, we compared the survival and cumulative event-free survival between both groups.

We evaluated patients' PS, defined as the conditional probability that a patient is assigned to the SAVR group given the patient's demographics, and measured the disease status and comorbidities. ${ }^{9}$ We selected 39 baseline covariates: age, sex, body mass index, body surface area, underweight, NYHA class III or IV, estimated glomerular filtration rate (eGFR), hypertension, diabetes mellitus, hyperlipidaemia, coronary artery disease, peripheral vascular disease, previous myocardial infarction, peripheral vascular disease, previous HF admission, prior heart surgery, chronic lung disease, malignancy, chronic liver disease, atrial fibrillation, haemodialysis treatment, peptic ulcer disease, limited activity, current smoker, aortic aneurysm or dissection, angiotensin-converting enzyme inhibitor, angiotensin II receptor blocker, calcium channel blocker, antiplatelet therapy, anticoagulant, $\beta$-blocker, corticosteroid, AVA, mean aortic pressure gradient, peak aortic velocity $\geq 4.0 \mathrm{~m} / \mathrm{s}$, left ventricular ejection fraction, aortic regurgitation, mitral regurgitation, tricuspid regurgitation and EuroSCORE II. Then, we matched non-SAVR and SAVR patients using PS and a greedy matching technique with a calliper of $0.2 \mathrm{SD}$ of the logit (PS). ${ }^{10}$ Furthermore, we assessed the balance of the baseline characteristics' distribution between the two groups by evaluating the standardised mean difference (SMD) as SMD $\leq 0.1$ suggests a balanced distribution of factors between the two groups. ${ }^{11}$ By performing subgroup analyses, we assessed the correlation between treatment and mortality and cardiovascular events in the groups of patients with prespecified risk factors at baseline of interests-advanced age ( $\geq 80$ years), asymptomatic status, renal insufficiency (eGFR $\leq 30 \mathrm{~mL} / \mathrm{min} / 1.73$ $\left.\mathrm{cm}^{2}\right)$ ) and a history of HF.

All statistical analyses were performed using STATA V.14 (STATA, College Station, Texas, USA) and R Statistical Software (V.3.3.2; R Foundation for Statistical
Computing, Vienna, Austria). Furthermore, we considered $\mathrm{p}<0.05$ (two-sided) as statistically significant.

\section{RESULTS}

\section{Clinical characteristics}

During the observation period up to June 2015, 210 patients finally underwent SAVR and 360 were medically managed. The median interval between the enrolment and SAVR date was 121 days (IQR: 35-624 days). Table 1 summarises the patients' baseline characteristics. At enrolment, the average age was 79 years, 236 were males $(41 \%), 314(55 \%)$ were symptomatic and $97(17 \%)$ presented severe daily symptoms (NYHA class III or IV). Regarding the AS severity assessments, SAVR patients exhibited a more severe valve status at the preoperative period concerning the AVA (non-SAVR vs SAVR, 0.76 vs $0.67 \mathrm{~cm}^{2}, \mathrm{p}<0.001$ ). For surgical intervention, bioprosthetic valves were more frequently used $(n=161$, $77 \%)$ than mechanical valves $(n=44,21 \%)$ and, notably, $49 \%$ of SAVR procedures were concomitant, including CABG (20\%), mitral valve replacement or repair $(24 \%)$, tricuspid valve replacement or repair $(8 \%)$ and ascending aorta replacement $(6 \%)$.

\section{Clinical outcomes}

During the follow-up (median 3.9 years; IQR, 1.8-5.2 years), $231(41 \%)$ patients died. The median follow-up period was 3.5 years (IQR, 1.2-5.2 years) for the non-SAVR group and 4.1 years (IQR, 2.7-5.1 years) for the SAVR group. Based on the Kaplan-Meier survival analysis (figure 2), SAVR patients exhibited 1-year, 3-year and 5-year estimated survival rates (from each operation date) of $91 \%, 87 \%$ and $84 \%$, respectively, compared with $81 \%, 57 \%$ and $43 \%$ in the non-SAVR group. Overall, survival and event-free survival rates were significantly higher in the SAVR group compared with the non-SAVR group during the follow-up period $(\mathrm{p}<0.001)$. Table 2 presents detailed clinical outcome data between the two groups. The rate of patients with $\mathrm{HF}$ admission was higher in the non-SAVR group within a 3-year period, whereas rates were similar during the complete follow-up period (non-SAVR, 38\% and SAVR, 36\%). We observed a gradual increment in the percentage of non-SAVR patients who developed syncope during the follow-up compared with $<1 \%$ of SAVR patients having syncope following SAVR.

\section{Clinical outcome and subgroup analysis in PS-matched patients}

The distribution of created PS is presented in figure 3A, and PS matching yielded 101 matched pairs of patients from both groups with a c-statistic of 0.90 . Furthermore, SMD was demonstrated in the unmatched and matched patients, highlighting that PS matching successfully decreased the SMD for 34 variables to an absolute value of $\leq 0.1$ (figure 3B). In the non-SAVR group, 101 patients exhibited a markedly lower survival and cumulative event-free survival than 101 patients in the SAVR group (figure 4). In addition, these risks were significantly 
Table 1 Baseline characteristics of the study patients according to the treatment

\begin{tabular}{|c|c|c|c|c|}
\hline & Entire cohort & Non-SAVR & SAVR & \\
\hline Variables & $\mathrm{N}=570$ & $\mathrm{~N}=360$ & $\mathrm{~N}=\mathbf{2 1 0}$ & P value* \\
\hline \multicolumn{5}{|l|}{ Clinical demographics } \\
\hline Age, years & $78.6(9.0)$ & $80.9(8.3)$ & $74.6(8.8)$ & $<0.001$ \\
\hline Age $>80$ years & $297(52)$ & $224(62)$ & $63(30)$ & $<0.001$ \\
\hline Male sex & $236(41)$ & $142(39)$ & $94(45)$ & 0.22 \\
\hline $\mathrm{BMI}, \mathrm{kg} / \mathrm{m}^{2}$ & $22.1(3.4)$ & $21.6(3.4)$ & $22.8(3.2)$ & $<0.001$ \\
\hline Underweight $\left(\mathrm{BMl}<18.5 \mathrm{~kg} / \mathrm{m}^{2}\right)$ & $83(15)$ & $72(20)$ & $11(5)$ & $<0.001$ \\
\hline $\mathrm{BSA}, \mathrm{m}^{2}$ & $1.47(0.17)$ & $1.44(0.16)$ & $1.51(0.18)$ & $<0.001$ \\
\hline \multicolumn{5}{|l|}{ Symptom at enrolment } \\
\hline NYHA class I & $256(45)$ & $167(46)$ & $61(29)$ & $<0.001$ \\
\hline NYHA class II & $217(38)$ & $121(34)$ & $123(59)$ & \\
\hline NYHA class III & $85(15)$ & $63(18)$ & $23(11)$ & \\
\hline NYHA class IV & $12(2)$ & $9(3)$ & $3(1)$ & \\
\hline Hypertension & $175(31)$ & $251(70)$ & $144(69)$ & 0.78 \\
\hline Diabetes & $155(27)$ & $95(26)$ & $60(29)$ & 0.63 \\
\hline Dyslipidaemia & $229(40)$ & $133(37)$ & $96(46)$ & 0.04 \\
\hline Hyperuricaemia & $63(11)$ & $40(11)$ & $23(11)$ & 1.00 \\
\hline Prior heart failure & $205(36)$ & $117(33)$ & $88(42)$ & 0.03 \\
\hline Coronary artery disease & $230(40)$ & $155(43)$ & $75(36)$ & 0.09 \\
\hline Old myocardial infarction & $78(14)$ & $60(17)$ & $18(9)$ & 0.01 \\
\hline Previous heart surgery & $100(18)$ & $73(20)$ & $27(13)$ & 0.03 \\
\hline eGFR $\left(\mathrm{mL} / \mathrm{min} / 1.73 \mathrm{~cm}^{2}\right)$ & $32(20)$ & $27(19)$ & $40(20)$ & $<0.001$ \\
\hline Renal insufficiency & $270(47)$ & $211(59)$ & $59(28)$ & $<0.001$ \\
\hline Haemodialysis & $69(12)$ & $49(14)$ & $20(10)$ & 0.18 \\
\hline Chronic lung disease & $61(11)$ & $39(11)$ & $22(11)$ & 1.00 \\
\hline Previous stroke & $77(14)$ & $54(15)$ & $24(11)$ & 0.26 \\
\hline Atrial fibrillation & $105(18)$ & $68(19)$ & $37(18)$ & 0.74 \\
\hline Peripheral vascular disease & $83(15)$ & $66(18)$ & $17(8)$ & 0.001 \\
\hline Aortic aneurysm or dissection & $40(7)$ & $27(8)$ & $13(6)$ & 0.61 \\
\hline Malignancy & $78(14)$ & $61(17)$ & $17(8)$ & 0.003 \\
\hline Chronic liver disease & $25(4)$ & $16(4)$ & $9(4)$ & 1.00 \\
\hline Peptic ulcer disease & $43(8)$ & $30(8)$ & $13(6)$ & 0.41 \\
\hline Limited activity & $110(19)$ & $103(29)$ & $7(3)$ & $<0.001$ \\
\hline Current smoker & $36(6)$ & $22(6)$ & $14(7)$ & 0.86 \\
\hline EuroSCORE II (\%)† & $3.3(2.0-5.4)$ & $3.8(2.6-6.2)$ & $2.3(1.5-3.9)$ & $<0.001$ \\
\hline STS score (PROM) (\%)† & $4.2(2.8-6.6)$ & $4.9(3.4-7.5)$ & $3.1(1.9-4.1)$ & $<0.001$ \\
\hline \multicolumn{5}{|l|}{ Medication use } \\
\hline Antiplatelet & $290(51)$ & $192(53)$ & $98(47)$ & 0.14 \\
\hline$\beta$-blocker & $144(25)$ & $88(24)$ & $56(27)$ & 0.55 \\
\hline ACE inhibitor or ARB & $324(57)$ & $145(40)$ & $101(48)$ & 0.08 \\
\hline Statins & $202(35)$ & $118(33)$ & $84(40)$ & 0.09 \\
\hline Calcium channel blockers & $246(43)$ & $165(46)$ & $81(39)$ & 0.10 \\
\hline Anticoagulant & $132(23)$ & $82(23)$ & $50(24)$ & 0.84 \\
\hline Corticosteroids & $14(3)$ & $11(3)$ & $3(1)$ & 0.27 \\
\hline
\end{tabular}


Table 1 Continued

\begin{tabular}{|c|c|c|c|c|}
\hline & Entire cohort & Non-SAVR & SAVR & \\
\hline Variables & $\mathrm{N}=570$ & $\mathrm{~N}=360$ & $\mathrm{~N}=\mathbf{2 1 0}$ & P value* \\
\hline Left ventricular ejection fraction (\%) & $61(12)$ & $60(13)$ & $62(11)$ & 0.04 \\
\hline Peak aortic velocity $(\mathrm{m} / \mathrm{s})$ & $3.7(0.9)$ & $3.4(0.9)$ & $4.3(0.8)$ & $<0.001$ \\
\hline Peak aortic velocity $>4.0 \mathrm{~m} / \mathrm{s}$ & $233(41)$ & $90(25)$ & $143(68)$ & $<0.001$ \\
\hline Aortic regurgitation (grade III-IV) & $35(6)$ & $17(5)$ & $18(9)$ & 0.14 \\
\hline Mitral regurgitation (grade III-IV) & $44(8)$ & $23(6)$ & $21(10)$ & 0.87 \\
\hline Tricuspid regurgitation (grade III-IV) & $44(8)$ & $28(9)$ & $16(9)$ & 1.00 \\
\hline \multicolumn{5}{|l|}{ Surgical procedure } \\
\hline Unknown & & \multicolumn{3}{|c|}{$5(2)$} \\
\hline Associated procedures, $n(\%)$ & & \multicolumn{3}{|c|}{$102(49)$} \\
\hline Coronary artery bypass grafting & & \multicolumn{3}{|c|}{$43(20)$} \\
\hline Mitral valve replacement or repair & & \multicolumn{3}{|c|}{$50(24)$} \\
\hline Tricuspid valve replacement or repair & & \multicolumn{3}{|c|}{$17(8)$} \\
\hline Ascending aorta replacement & & \multicolumn{3}{|c|}{$13(6)$} \\
\hline
\end{tabular}

${ }^{*} \mathrm{P}$ value refers to comparisons between the non-SAVR group at enrolment and the SAVR group at operation.

†Median and IQR. Data are mean (SD) or $\mathrm{n}(\%)$.

ACEl, angiotensin-converting enzyme inhibitor; ARB, angiotensin II receptor blocker; BMI, body mass index; BSA, body surface area; NYHA class, New York Heart Association functional classification; PROM, predicted risk of mortality; SAVR, surgical aortic valve replacement; STS,

Society of Thoracic Surgery; eGFR, estimated glomerular filtration rate.

higher in the non-SAVR group compared with the SAVR group (HR $0.35,95 \%$ CI 0.21 to 0.59 , $\mathrm{p}<0.001$, for all-cause mortality; HR $0.53,95 \%$ CI 0.34 to $0.82, \mathrm{p}=0.004$, for $\mathrm{HF}$ hospitalisation and mortality; HR $0.62,95 \%$ CI 0.42 to $0.92, \mathrm{p}=0.02$, for cardiovascular events and mortality).

We performed a subgroup analysis for the PS-matched population (table 3 ). Patients aged $\geq 80$ years (non-SAVR vs SAVR: HR $0.35 ; 95 \%$ CI 0.21 to $0.34 ; \mathrm{p}<0.001$ ), with cardiac symptoms (NYHA class II-IV; HR 0.19; $95 \%$ CI 0.10 to $0.37 ; \mathrm{p}<0.001)$ or a history of admission due to HF (HR 0.38; 95\% CI 0.19 to 0.78; $\mathrm{p}=0.009$ ) exhibited a significant correlation with the survival benefit during
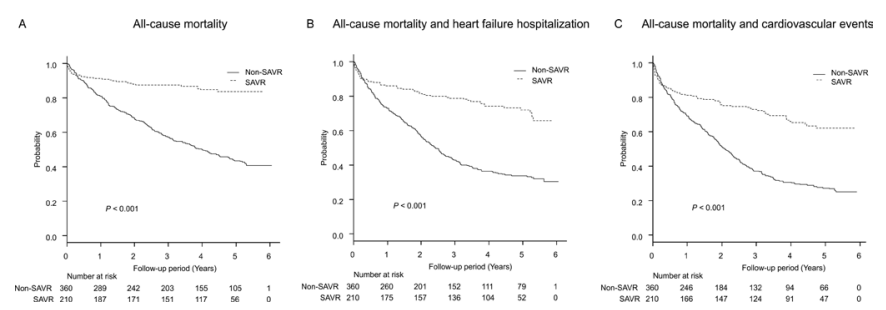

Figure 2 Cumulative event-free survival curves between the non-SAVR $(n=360)$ and SAVR $(n=210)$ groups in the entire population regarding (A) all-cause mortality, (B) all-cause mortality and heart failure hospitalisation and (C) all-cause mortality and cardiovascular events. SAVR, surgical aortic valve replacement. the follow-up period, although it was not significant in the population without cardiac symptoms (HR 0.83; 95\% CI 0.38 to $1.84 ; \mathrm{p}=0.66$ ) or with renal insufficiency (HR $0.60 ; 95 \%$ CI 0.33 to $1.07 ; p=0.09)$.

\section{DISCUSSION}

This study established that SAVR markedly correlated with the survival improvement and prevention of major cardiovascular events compared with medical treatments during the follow-up period in both the entire severe AS and PS-matched populations. In a few recent studies, researchers attempted to more precisely investigate the treatment effect of SAVR on long-term outcome within a well-balanced sample. Pai et al reported that SAVR correlated with a survival benefit in three of the four strata produced by the PS stratification method. ${ }^{12}$ Their findings are consistent with those of the present study, although approximately $55 \%$ of our population was symptomatic and there was a delay between the diagnosis and SAVR along with a possible baseline characteristic imbalance between the two groups. In addition, we observed that the overall survival rates of both groups were higher than a prior study in which the 1-year, 2-year and 5-year survival rates among 277 patients with severe AS were $87 \%, 78 \%$ and $68 \%$ in the SAVR group and $52 \%, 40 \%$ 
Table 2 Yearly rates according to clinical events during the follow-up period between the non-SAVR $(n=360)$ and SAVR $(n=210)$ groups

Time (years)

\begin{tabular}{|c|c|c|c|c|c|c|c|}
\hline Clinical events & & $1(\%)$ & $2(\%)$ & $3(\%)$ & Total in group (\%) & Total & $\begin{array}{l}\text { P } \\
\text { value* }\end{array}$ \\
\hline \multirow[t]{2}{*}{ All-cause death } & Non-SAVR & 18.9 & 31.7 & 42.5 & 55.6 & $40.5 \%$ & $<0.001$ \\
\hline & SAVR & 8.6 & 11.9 & 12.4 & 14.8 & & \\
\hline \multirow[t]{2}{*}{ HF admission } & Non-SAVR & 15.6 & 24.7 & 32.8 & 37.8 & $37.0 \%$ & $<0.001$ \\
\hline & SAVR & 5.7 & 7.1 & 8.6 & 35.7 & & \\
\hline \multirow[t]{2}{*}{ Nonfatal stroke } & Non-SAVR & 2.5 & 6.1 & 7.5 & 9.7 & $8.6 \%$ & 0.49 \\
\hline & SAVR & 3.3 & 4.3 & 5.7 & 6.7 & & \\
\hline \multirow[t]{2}{*}{ Syncope } & Non-SAVR & 1.7 & 2.8 & 4.2 & 5.0 & $3.3 \%$ & 0.002 \\
\hline & SAVR & 0.0 & 0.0 & 0.0 & 0.5 & & \\
\hline \multirow[t]{2}{*}{ ACS } & Non-SAVR & 0.8 & 0.8 & 1.4 & 1.4 & $1.4 \%$ & 0.42 \\
\hline & SAVR & 0.5 & 0.5 & 0.5 & 1.4 & & \\
\hline
\end{tabular}

${ }^{*} \mathrm{P}$ value refers to comparisons of event rates between the non-SAVR and SAVR groups at 3 years. Data are \% values.

ACS, acute coronary syndrome; HF, heart failure; SAVR, surgical aortic valve replacement.

and $22 \%$ in the non-SAVR group, respectively; notably, their population comprised an older population (average age, 85 years) and more advanced stenosis (defined as AVA $\left.\leq 0.8 \mathrm{~cm}^{2}\right) .{ }^{13}$ Bach and his colleagues reported that the mortality rate of symptomatic patients with severe AS and relatively low-operative risk based on the EuroSCORE II who did not undergo SAVR was $53 \%$ at 3 years. ${ }^{14}$ The long-term outcome in this study seemed more reassuring at the 3-year mark, with a mortality of approximately $17 \%$ in the SAVR group. Regarding short-term mortality, SAVR-related operative risks have declined over the last two decades, although patients receiving SAVR are now much older and with a higher prevalence of comorbidities. ${ }^{15}$ Studies have estimated a 30 -day mortality of $<3 \%$ for isolated SAVR and $<4.5 \%$ for combined SAVR and CABG. ${ }^{16}$ Our study obtained a remarkable achievement rate for 30-day mortality as only $5(2 \%)$ of 210 patients died within 30 days of SAVR, in which $49 \%$ of SAVR patients underwent concomitant surgical procedures, such as mitral valve replacement or CABG.

Limited data are currently available on detailed clinical events in patients with severe AS, especially among those who underwent SAVR. ${ }^{17}$ Our study elucidated the breakdown of cardiovascular events during the follow-up period less optimistically. In fact, admission for HF was the leading adverse event in both groups and was markedly different at 3 years. However, during the entire follow-up period, the admission rate for HF in the SAVR group reached $36 \%$, which corroborated the non-SAVR group $(38 \%)$. The findings of a few previous studies ${ }^{12}$ are consistent with this study, although almost $55 \%$ of our population was symptomatic and there was a delay between the diagnosis and SAVR along with a possible baseline characteristics imbalance between the two groups. These remarkable findings of high readmission rates in SAVR patients may be explained by two primary factors: increases in valve-related events and established myocardial damage affecting postoperative outcomes. There are clinical issues with valve deterioration and durability and increased risk of valve-related events such as prosthetic valve endocarditis and thrombosis. Andrew and colleagues ${ }^{18}$ reported that patients with AS with severe diastolic dysfunction have more diffuse myocardial fibrosis detected on equilibrium contrast cardiovascular MRI, indicating that the regression of left ventricular hypertrophy 6 months following SAVR is due to cellular rather than fibrosis resolution. Therefore, we can speculate that a complete reversible change in the cardiac function before and after SAVR cannot be expected in patients with AS in whom myocardial damage has completely developed. Further studies are warranted to explore the potential mechanisms of $\mathrm{HF}$ in patients undergoing SAVR.

Additionally, our subgroup analysis with created PS-matched samples added new findings that these positive correlations remained substantial among patients aged $\geq 80$ years, even in symptomatic patients or those with a history of HF admission. Likewise, this study revealed that a correlation between SAVR and survival decreased among asymptomatic patients and those with renal insufficiency. It is typically accepted from the current guidelines that the symptom development is a vital indication for intervention, whether surgical or transcatheter. A recent comprehensive study using the Japanese population recommended early intervention in selected asymptomatic subjects with AS because long-term outcomes of symptomatic patients were worse than those of asymptomatic patients. ${ }^{19}$ In fact, some groups of patients with AS processed the decompensation without any complaints at daily practice because of frailty, cognitive disorder or workload avoidance. Of note, an appropriate assessment of the symptomatic status can be challenging, especially 
A

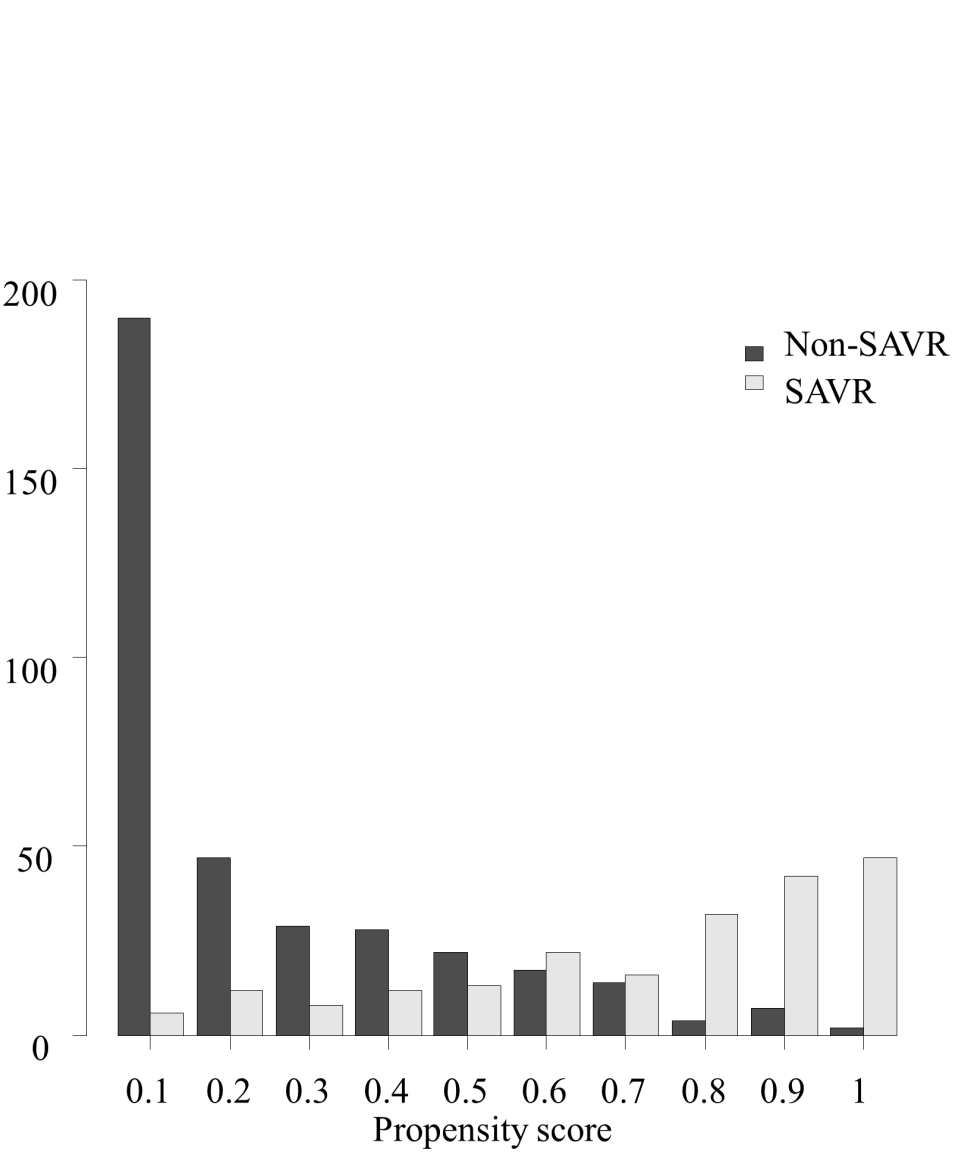

B

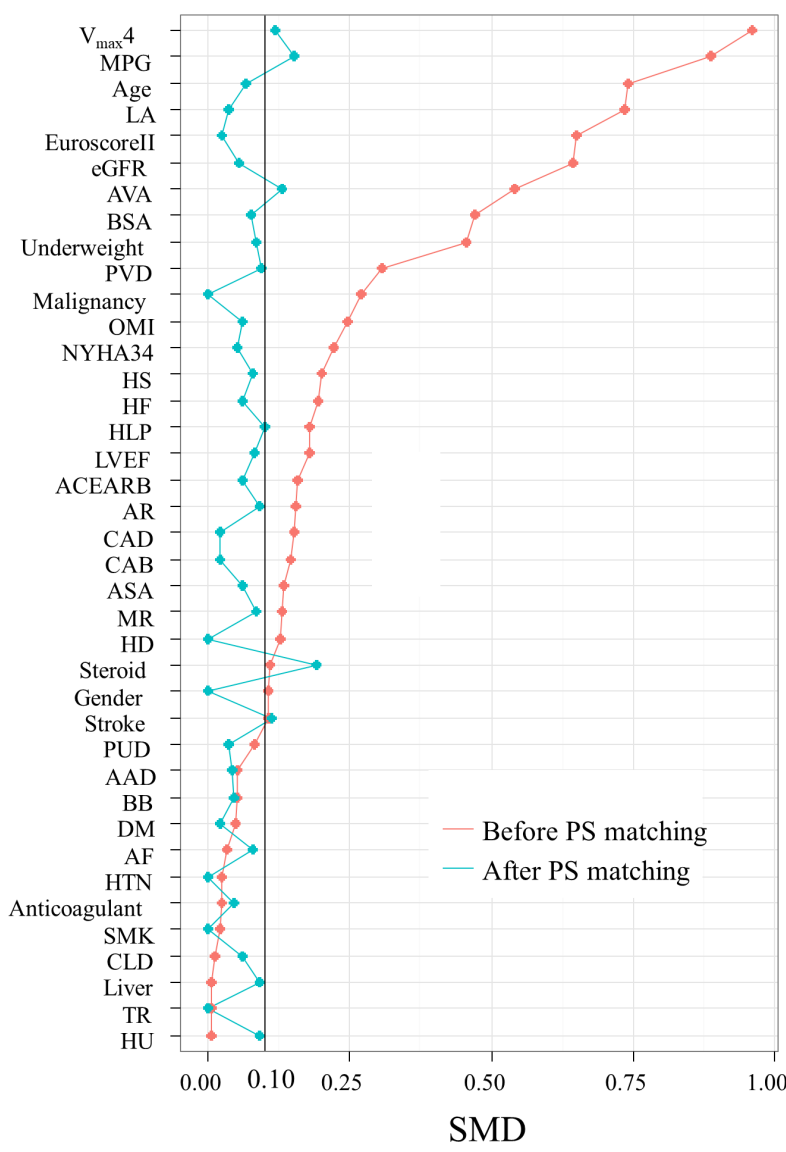

Figure 3 Distributions of PS between the non-SAVR $(n=360)$ and SAVR $(n=210)$ groups $(A)$. The SMD of the 39 selected baseline variables before and after PS matching (B). AAD, aortic aneurysm or dissection; ACEI, angiotensin-converting enzyme inhibitor; ARB, angiotensin II receptor blocker; AF, atrial fibrillation; AR, aortic regurgitation; ASA, antiplatelet; AVA, aortic valve area; $\mathrm{BB}, \beta$-blocker; $\mathrm{BMI}$, body mass index; $\mathrm{BSA}$, body surface area; $\mathrm{CAB}$, calcium channel blocker; $\mathrm{CAD}$, coronary artery disease; CLD, chronic lung disease; DM, diabetes mellitus; eGFR, estimated glomerular filtration rate; HD, haemodialysis; HTN, hypertension; HLP, hyperlipidaemia; HF, heart failure; HS, prior history of cardiac surgery, HU, hyperuricaemia; LA, limited activity; LVEF, left ventricular ejection fraction; MPG, mean aortic gradient; MR, mitral regurgitation; NYHA34, New York Heart Association class III or IV; OMI, old myocardial infarction; PVD, peripheral vascular disease; PS, propensity score; PUD, peptic ulcer disease; SAVR, surgical aortic valve replacement; SMD, standardised mean differences; SMK, current smoker; TR, tricuspid regurgitation; $\mathrm{V}_{\max } 4$, peak aortic velocity $>4.0 \mathrm{~m} / \mathrm{s}$.

in elderly patients who commonly have impaired physical mobility and cognitive impairment. ${ }^{20}$ Thus, a systematic strategy to assess symptoms among elderly patients with AS is warranted to avoid underestimation. Moreover,

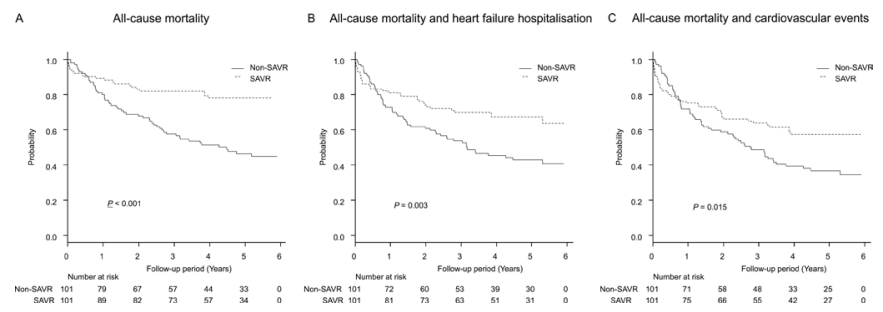

Figure 4 Cumulative event-free survival curves between the non-SAVR $(n=101)$ and SAVR $(n=101)$ groups in the PSmatched population regarding $(A)$ all-cause mortality, $(B)$ all-cause mortality and heart failure hospitalisation and (C) all-cause mortality and cardiovascular events. PS, propensity score; SAVR, surgical aortic valve replacement. some studies reported that patients with AS and preoperative renal dysfunction exhibited an increased operative risk for SAVR, ${ }^{21}$ with acute kidney injury following SAVR occurring in $3.4 \%-43 \%$ of SAVR cases. Reportedly, the 30-day mortality for these patients was $5.5 \%-46 \% .^{22}$ In patients with pre-existing renal dysfunction, valve replacement with mechanical prostheses poses a higher risk for anticoagulation-related complications, whereas bioprosthetic replacement might be complicated by acceleration in structural valve deterioration. ${ }^{23}$ Hence, further studies are warranted to elucidate the appropriate indications, methods and timing of intervention for patients with severe AS and renal dysfunction.

\section{Study limitations}

This relatively large cohort study holds several limitations. First, selection bias should be considered in this non-randomised study which could account for massive 
Table 3 Correlation between SAVR and the three types of clinical events in the selected subgroups of the propensity scorematched population

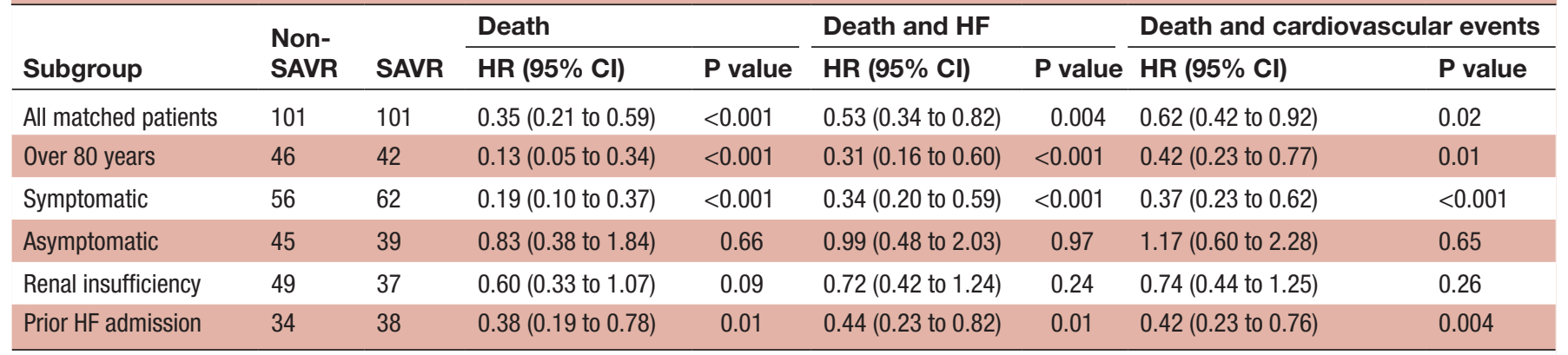

HF, heart failure; SAVR, surgical aortic valve replacement.

confounding by indication between both groups. Thus, besides a crude comparison, we applied the PS-mediated analysis to adjust for selection bias and potential confounding factors rigorously, although a room still remains for discussion regarding the variable selection and manipulation for a fairer comparison of the process of obtaining these findings. Second, the matching method, while attaining a more well-balanced matching, decreased the matched sample population by almost $50 \%$ in the SAVR group, that might affect its generalisability. Finally, being a single-centre, retrospective, observational study, our findings might, therefore, not be generalised to other facilities within or outside of Japan. However, our heart centre is the only largest cardiovascular facility in the area, and almost all patients with diseased valves potentially warranting surgical intervention are referred to us for the comprehensive examination and follow-up without much selection. Consequently, a relatively large number of patients with severe AS were successfully reviewed in a short period along with comprehensive records of patients' characteristics.

\section{CONCLUSION}

This study establishes a correlation between SAVR and a lower incidence of both all-cause mortality and adverse cardiovascular events during the total follow-up period of 3.9 years in the 570 patients with severe AS and the PS-matched population. The patients with severe AS undergoing SAVR and concomitant surgical procedures were related to a $65 \%$ risk reduction of mortality and $38 \%$ risk reduction of adverse cardiovascular events compared with the medically treated cohort following rigorous adjustment for baseline characteristics. Notwithstanding, sufficient room exists to consider surgical indications, procedure types and timing for severe AS in asymptomatic patients. These findings have significant implications for informed decision-making during the management of patients with severe AS.

Acknowledgements The authors would like to thank Dr Alan Kimber for his guidance and interest in this study along with the continued support of the staff from Faculty of Social, Human and Mathematical Sciences in the University of Southampton.
Contributors SM had full access to all the data in the study and takes responsibility for the integrity of the data and the accuracy of the data analysis. Acquisition, analysis or interpretation of data: all authors. Drafting of the manuscript: SM. Critical revision of the manuscript for important intellectual content: HK, TY. Administrative, technical or material support: SS, MH. Study supervision: KA.

Funding The authors have not declared a specific grant for this research from any funding agency in the public, commercial or not-for-profit sectors.

Competing interests HK is affiliated with the Department of Healthcare Quality Assessment at the University of Tokyo, a social collaboration department supported by the National Clinical Database, Johnson \& Johnson K.K. and Nipro Co. The remaining authors have no conflicts of interest to declare.

Patient consent for publication Not required.

Provenance and peer review Not commissioned; externally peer reviewed.

Data availability statement All data relevant to the study are included in the article or uploaded as supplementary information.

Open access This is an open access article distributed in accordance with the Creative Commons Attribution Non Commercial (CC BY-NC 4.0) license, which permits others to distribute, remix, adapt, build upon this work non-commercially, and license their derivative works on different terms, provided the original work is properly cited, appropriate credit is given, any changes made indicated, and the use is non-commercial. See: http://creativecommons.org/licenses/by-nc/4.0/.

\section{REFERENCES}

1. Lindroos M, Kupari M, Heikkilä J, et al. Prevalence of aortic valve abnormalities in the elderly: an echocardiographic study of a random population sample. J Am Coll Cardiol 1993;21:1220-5.

2. Dweck MR, Boon NA, Newby DE. Calcific aortic stenosis: a disease of the valve and the myocardium. J Am Coll Cardiol 2012;60:1854-63.

3. Matsumura T, Ohtaki E, Misu K, et al. Etiology of aortic valve disease and recent changes in Japan:a study of 600 valve replacement cases. Int J Cardiol 2002;86:217-23.

4. Miura S, Arita T, Kumamaru $\mathrm{H}$, et al. Causes of death and mortality and evaluation of prognostic factors in patients with severe aortic stenosis in an aging Society. $J$ Cardiol 2015;65:353-9.

5. Leon MB, Smith CR, Mack MJ, et al. Transcatheter or surgical aortic-valve replacement in intermediate-risk patients. N Engl J Med 2016;374:1609-20.

6. Bonow RO, Carabello BA, Chatterjee $\mathrm{K}$, et al. Focused update incorporated into the ACC/AHA 2006 guidelines for the management of patients with valvular heart disease: a report of the American College of cardiology American Heart Association Task Force on practice guidelines (writing Committee to revise the 1998 guidelines for the management of patients with valvular heart disease): endorsed by the Society of cardiovascular Anesthesiologists, Society for cardiovascular angiography and interventions, and Society of thoracic surgeons. J Am Coll Cardiol 2008;2008:E1-142.

7. Oh JK, Taliercio CP, Holmes DR, et al. Prediction of the severity of aortic stenosis by Doppler aortic valve area determination: prospective Doppler-catheterization correlation in 100 patients. J Am Coll Cardiol 1988;11:1227-34. 
8. Bonow RO, Carabello BA, Kanu C, et al. ACC/AHA 2006 guidelines for the management of patients with valvular heart disease: a report of the American College of Cardiology/American Heart Association Task Force on practice guidelines (writing Committee to revise the 1998 guidelines for the management of patients with valvular heart disease): developed in collaboration with the Society of cardiovascular Anesthesiologists: endorsed by the Society for cardiovascular angiography and interventions and the Society of thoracic surgeons. Circulation 2006;114:e84-231.

9. Horwitz RI, Viscoli CM, Clemens JD, et al. Developing improved observational methods for evaluating therapeutic effectiveness. Am J Med 1990;89:630-8.

10. Austin PC. Optimal caliper widths for propensity-score matching when estimating differences in means and differences in proportions in observational studies. Pharm Stat 2011;10:150-61.

11. ------ . The relative ability of different propensity score methods to balance measured covariates between treated and untreated subjects in observational studies. Med Decis Making 2009;29:661-77.

12. Pai RG, Kapoor N, Bansal RC, et al. Malignant natural history of asymptomatic severe aortic stenosis: benefit of aortic valve replacement. Ann Thorac Surg 2006;82:2116-22.

13. Varadarajan $\mathrm{P}, \mathrm{Kapoor} \mathrm{N}$, Bansal RC, et al. Survival in elderly patients with severe aortic stenosis is dramatically improved by aortic valve replacement: Results from a cohort of 277 patients aged $>$ or $=80$ years. Eur J Cardiothorac Surg 2006;30:722-7.

14. Bach DS, Siao D, Girard SE, et al. Evaluation of patients with severe symptomatic aortic stenosis who do not undergo aortic valve replacement: the potential role of subjectively overestimated operative risk. Circ Cardiovasc Qual Outcomes 2009;2:533-9.
15. Lee R, Li S, Rankin JS, et al. Fifteen-year outcome trends for valve surgery in North America. Ann Thorac Surg 2011;91:677-84.

16. Brown JM, O'Brien SM, Wu C, et al. Isolated aortic valve replacement in North America comprising 108,687 patients in 10 years: changes in risks, valve types, and outcomes in the Society of thoracic surgeons national database. J Thorac Cardiovasc Surg 2009;137:82-90.

17. Auensen A, Hussain Al, Bendz B, et al. Morbidity outcomes after surgical aortic valve replacement. Open Heart 2017;4:e000588

18. Flett AS, Sado DM, Quarta G, et al. Diffuse myocardial fibrosis in severe aortic stenosis: an equilibrium contrast cardiovascular magnetic resonance study. Eur Heart J Cardiovasc Imaging 2012;13:819-26.

19. Shirai S, Taniguchi T, Morimoto T, et al. Five-year clinical outcome of asymptomatic vs. symptomatic severe aortic stenosis after aortic valve replacement. Circ J 2017;81:485-94.

20. Zilberszac R, Gabriel H, Schemper M, et al. Asymptomatic severe aortic stenosis in the elderly. JACC Cardiovasc Imaging 2017; 10:43-50

21. Thourani VH, Keeling WB, Sarin EL, et al. Impact of preoperative renal dysfunction on long-term survival for patients undergoing aortic valve replacement. Ann Thorac Surg 2011;91:1798-807.

22. Najjar M, Salna M, George I. Acute kidney injury after aortic valve replacement: incidence, risk factors and outcomes. Expert Rev Cardiovasc Ther 2015;13:301-16.

23. Ju MH, Jung S-H, Choo SJ, et al. Valve replacement surgery in severe chronic kidney disease. Int J Cardiol 2017;241:115-9. 